\title{
On the electronic structure of alternant conjugated organic radicals for light-emitting diode applications
}

Hele, Timothy

Timothy J. H. Hele, "On the electronic structure of alternant conjugated organic radicals for light-emitting diode applications," Proc. SPIE 11799, Physical Chemistry of Semiconductor Materials and Interfaces XX, 117991A (26 August 2021); doi: 10.1117/12.2593712

SPIE Event: SPIE Nanoscience + Engineering, 2021, San Diego, California, United States 


\title{
On the electronic structure of alternant conjugated organic radicals for light-emitting diode applications
}

\author{
Timothy J. H. Hele ${ }^{\mathrm{a}}$ \\ a Department of Chemistry, University College London, 20 Gordon Street, WC1H 0AJ, \\ London, United Kingdom
}

\begin{abstract}
Since the demonstration in 2018 that organic radicals can be used to make highly efficient organic light-emitting diodes, there has been an explosion of interest in their capabilities and many experimental and computational studies of their performance. Here we take a theoretical view and describe the electronic structure of radicals from an algebraic perspective. By rediscovering and adapting historic investigations of organic radicals, we show how many experimentally useful properties can be determined without synthesis or computation, but simply from knowledge of the molecular structure and in particular whether or not the radical is an alternant hydrocarbon. These include the location of orbital amplitude in the SOMO, absorption and emission characteristics, and solvatochromic shift. We explain these results in the context of modern organic light-emitting diodes in order to inform future investigations.
\end{abstract}

\section{INTRODUCTION}

In 2018 a record-breaking organic light-emitting diode (OLED) was made with $27.6 \%$ external quantum efficiency (EQE) and approximately 100\% internal quantum efficiency (IQE). ${ }^{1}$ Unlike most organic light-emitting diodes, this was not based on a closed-shell molecule but on an organic radical, TTM-3NCz [tris-(2,4,6-trichlorophenyl)methyl 3-substituted-9-(naphthalen-2-yl)-9H-carbazole]. ${ }^{1}$ Radicals (strictly speaking, monoradicals ${ }^{*}$ ) are molecules with one unpaired electron whose qualitative electronic structure is shown in Fig. 1.

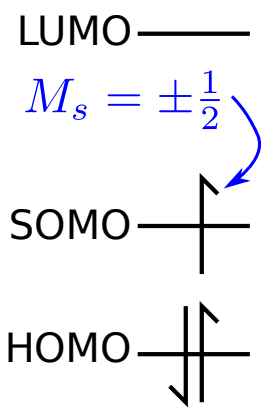

Figure 1. Qualitative electronic structure of a radical, with a filled Highest Occupied Molecular Orbital (HOMO), half-filled Singly Occupied Molecular Orbital (SOMO), and empty Lowest Unoccupied Molecular Orbital (LUMO).

The internal quantum efficiency of conventional closed-shell molecules is usually limited to $25 \%$ by the spinstatistics limit, but there is no such restriction for radicals. ${ }^{2,3}$ Historically, radical molecules were often considered too unstable ${ }^{4,5}$ for serious consideration in OLEDs, and those that were stable (such as the triphenylmethyl radical and its derivatives) were usually non-emissive, leading to the view of radical OLEDs as somewhat of an obscure intellectual curiosity. However, in 2015 Peng et al. demonstrated an OLED based on an organic radical, TTM-1Cz [(4-N-carbazolyl-2,6-dichlorophenyl)bis(2,4,6-trichlorophe- nyl)methyl radical]. ${ }^{6}$ This was, to our knowledge, the first demonstration of a functioning radical OLED, with EQE of $2.4 \%{ }^{6}$

Email: t.hele@ucl.ac.uk

*Monoradicals have only one unpaired electron. Radicals with two or more unpaired electrons have not, to our knowledge, been used in functioning OLEDs and are not considered in this article.

Physical Chemistry of Semiconductor Materials and Interfaces XX, edited by Andrew J. Musser, Derya Baran, Daniel Congreve, Christian Nielsen, Proc. of SPIE Vol. 11799, 117991A

(C) 2021 SPIE · CCC code: 0277-786X/21/\$21 - doi: 10.1117/12.2593712

Proc. of SPIE Vol. 11799 117991A-1 
Since the discovery in 2018 of a highly-efficient radical OLED, there has been great interest in radical optoelectronics, both synthetic ${ }^{2,5}$ and computational, ${ }^{5,7-10}$ as well as review articles from an organic synthetic perspective $^{3}$ and an applied physics viewpoint. ${ }^{11}$ However, there has been comparatively little research into the theoretical electronic structure of organic radicals, and the success of methods such as Hückel theory and Pariser-Parr-Pople (PPP) theory ${ }^{12-15}$ for closed-shell optoelectronic species ${ }^{16-19}$ suggests that this could be a promising avenue of research for radicals too. We therefore seek to go beyond the qualitiative (but usually correct) depiction of radical electronic structure as shown in Fig. 1.

To the best of our knowledge, the electronic structure of radicals was first investigated theoretically by Longuet-Higgins in $1950,{ }^{20}$ then by Dewar and Longuet-Higgins in $1954^{21}$ and in more detail be Longuet-Higgins and Pople in $1955 .^{22}$ These last two articles were part of a series of articles on the electronic structure of conjugated organic species ${ }^{15,21-24}$ and built on earlier work such as the Coulson-Rushbrooke theorem ${ }^{25}$ and its application to radicals by Longuet-Higgins. ${ }^{20}$ As far as we are aware these articles were not known to the optoelectronic field until they were rediscovered in 2019 and their results used to inform and predict the design of radical OLEDs in an article published in $2020 .^{2}$ At the time Dewar, Longuet-Higgins and Pople wrote these articles electronic structure theory notation was not standardised and the standard notation used today ${ }^{26}$ is different in many respects that that used by them. There is therefore a need to rederive their results in modern notation such that they may be more easily appreciated by the modern optoelectronics community.

In this article we therefore seek to rederive the main results from the papers by Dewar, Longuet-Higgins and Pople ${ }^{21,22}$ concerning neutral organic radicals, and interpret their results in the context of modern organic light-emitting diode design. We are also able, in some circumstances, to derive results which are more general and widely-applicable than those originally presented.

We stress that there are many other theoretical techniques which may be of use in explaining the functionality of radical OLEDs, and which have previously been successfully applied in optoelectronic systems, such as group theory ${ }^{27}$ which can inform orbital mixing ${ }^{16,19,28}$ and spin-orbit coupling, ${ }^{28,29}$ as well as intensity borrowing theory. ${ }^{2,16,30}$ Further application of these theories to radicals is left as future work.

The article is structured as follows. In section 2 we detail spin considerations, followed by choosing the molecular orbitals to optimize (lower) the energy of the ground state in section 3 and the computation of excited states in section 4. In section 5 we introduce an atomic orbital basis and in section 6 we apply the standard Pariser-Parr-Pople approximations. In section 7 we see how, for the case of an alternant hydrocarbon radical, substantial simplifications occur and derive various molecular properties. Application to OLEDs are considered in section 8 before conclusions are given in section 9 .

\section{SPIN CONSIDERATIONS}

In this article we consider a molecule with $N_{e}$ electrons where $N_{e}$ is odd, such that (in a restricted wavefunction) there will be $k=\left(N_{e}-1\right) / 2$ paired electrons and one unpaired electron. For reasons which will become apparent later, and in accordance with later convention, ${ }^{13,16}$ we number the SOMO orbital zero, and the HOMO, HOMO -1 etc as $1,2, \ldots$ while the LUMO, LUMO +1 etc are numbered $1^{\prime}, 2^{\prime}, \ldots$ We also assume that the unpaired electron is in an $\alpha$ (up spin) orbital.

One common way of describing a radical system is with an unrestricted wavefunction, ${ }^{22,26}$ i.e.

$$
\left|\Psi_{0}^{u}\right\rangle=\left|\psi_{k}^{\alpha} \bar{\psi}_{k}^{\beta} \psi_{k-1}^{\alpha} \bar{\psi}_{k-1}^{\beta} \ldots \psi_{1}^{\alpha} \bar{\psi}_{1}^{\beta} \psi_{0}^{\alpha}\right\rangle
$$

where $\psi_{k}^{\alpha}$ is the wavefunction for the $k$ th up spin orbital and $\bar{\psi}_{k}^{\beta}$ the wavefunction for the $k$ th down spin orbital, and the spatial parts of $\psi_{k}^{\alpha}$ and $\bar{\psi}_{k}^{\beta}$ are not required to be the same. The bar in $\bar{\psi}_{k}^{\beta}$ denotes that the spin part of the wavefunction is down spin $\left(m_{s}=-1 / 2\right)$. This description, commonly used in Unrestricted Hartree-Fock (UHF) and many Density Functional Theory (DFT) calculations, ${ }^{1,2}$ can provide a good description of the ground state but struggles to accurately describe higher-lying excited states due to spin-contamination. ${ }^{7}$

This is because, in nonrelativistic systems which we consider here, eigenstates of the electronic Hamiltonian $\hat{H}$ must also be eigenstates of the spin projection operator $\hat{S}_{z}$ (with eigenvalue $M_{s}$, setting $\hbar=1$ ) and the total spin operator $\hat{S}^{2}$ (with eigenvalue $S(S+1)$. The wavefunction in Eq. (1) is an eigenstate of $\hat{S}_{z}$ with eigenvalue 
$+1 / 2$, but is not usually an eigenstate of $\hat{S}^{2}{ }^{26}$ This means that, especially when there are multiple unpaired electrons, it is not possible to determine whether the state is a doublet or a quartet, sometimes referred to as the spin-contamination problem. ${ }^{7}$ The lack of clear doublet/quartet character is highly problematic when comparing computational results to experimental measurements, since optical transitions are only permitted between states of the same spin, which in the case of radicals means between different doublet states or between different quartet states, but not from doublets to quartets or vice versa.

The difficulty of accurately describing the excited states of radicals with unrestricted calculations was, to our knowledge, first explained in 1955. ${ }^{22}$ This was rediscovered by $\mathrm{He}$ et al. in $2019^{7}$ where computational calculations confirmed Longuet-Higgins' theoretical predictions.

Instead of the unrestricted wavefunction, Longuet-Higgins et al. suggest using a restricted one: ${ }^{22}$

$$
\left|\Psi_{0}\right\rangle=\left|\psi_{k} \bar{\psi}_{k} \psi_{k-1} \bar{\psi}_{k-1} \ldots \psi_{1} \bar{\psi}_{1} \psi_{0}\right\rangle
$$

where the spatial part of $\psi_{k}$ and $\bar{\psi}_{k}$ are the same, such that the $\alpha$ and $\beta$ superscripts can be omitted. This can be achieved through restricted open-shell Hartree Fock (ROHF) calculations, explicitly spin-adapting DFT (sometimes referred to as X-TDDFT ${ }^{7}$ ) and through high-level complete active space self-consistent field (CASSCF) calculations.

The wavefunction in Eq. (2) is an eigenstate of $\hat{S}^{2}$ with an eigenvalue 3/4, corresponding to a doublet. Excitedstate wavefunctions based on Eq. (2) may not necessarily be eigenstates of $\hat{S}^{2}$ but simple linear combinations of excited states can be found which are eigenstates of $\hat{S}^{2} .{ }^{22}$

\section{CHOICE OF MOLECULAR ORBITALS}

Having determined that we are to consider a restricted wavefunction, we now discuss how best to calculate the spatial part of the molecular orbitals. There are, of course, many different approaches and here we will follow the one of Longuet-Higgins and Pople. ${ }^{22}$ They begin, as with the conventional derivation of the Roothaan equations, ${ }^{26}$ by defining the optimal orbitals to be those which minimize the energy of the (restricted) ground state determinant $\left|\Psi_{0}\right\rangle$ of Eq. (2). Any infinitestimal variation in the orbitals of $\left|\Psi_{0}\right\rangle$ is equivalent to incorporating small amounts of singly excited configurations:

$$
\left|\Psi_{0}\right\rangle \rightarrow\left|\Psi_{0}\right\rangle+\sum_{i=1}^{k} \lambda_{i 0}\left|\Psi_{\bar{i}}^{\overline{0}}\right\rangle+\sum_{j=1}^{\infty} \lambda_{0 j}\left|\Psi_{0}^{j^{\prime}}\right\rangle+\sum_{i=1}^{k} \sum_{j=1}^{\infty} \lambda_{i j}\left(\left|\Psi_{i}^{j^{\prime}}\right\rangle+\left|\Psi_{\bar{i}}^{\bar{j}^{\prime}}\right\rangle\right)
$$

where $\left|\Psi_{i}^{j^{\prime}}\right\rangle$ corresponds to exciting an electron from spin orbital $i$ to (unoccupied) spin orbital $j^{\prime}$. The second term on the RHS of Eq. (3) corresponds to exciting an electron into the empty SOMO spin-down orbital, the third term to exciting the up-spin electron in the SOMO to an empty orbital $j^{\prime}$ and the fourth term to an in-phase combination of exciting from occupied orbital $i$ to unoccupied orbital $j^{\prime}$. These three terms are all eigenstates of $\hat{S}^{2}$ and with eigenvalue $3 / 4$ (setting $\hbar=1$ ), i.e. they are doublets, and for simplicity we define the normalized state

$$
\left|\Psi_{i}^{j^{\prime},+}\right\rangle=\frac{1}{\sqrt{2}}\left(\left|\Psi_{i}^{j^{\prime}}\right\rangle+\left|\Psi_{\bar{i}}^{\bar{j}^{\prime}}\right\rangle\right)
$$

These excitations are illustrated in Fig. 2.

Small perturbations of the ground state could also mix in the states containing $\left|\Psi_{\bar{i}}^{\bar{j}^{\prime}}\right\rangle-\left|\Psi_{i}^{j^{\prime}}\right\rangle$; this linear combination is not an eigenstate of $\hat{S}^{2}$ and is not considered further.

The change in energy associated with Eq. (3) is therefore

$$
\delta E=\sum_{i=1}^{k} \lambda_{i 0}\left\langle\Psi_{0}|\hat{H}| \Psi_{\bar{i}}^{\overline{0}}\right\rangle+\sum_{j=1}^{\infty} \lambda_{0 j}\left\langle\Psi_{0}|\hat{H}| \Psi_{0}^{j^{\prime}}\right\rangle+\sum_{i=1}^{k} \sum_{j=1}^{\infty} \sqrt{2} \lambda_{i j}\left\langle\Psi_{0}|\hat{H}| \Psi_{i}^{j^{\prime},+}\right\rangle .
$$




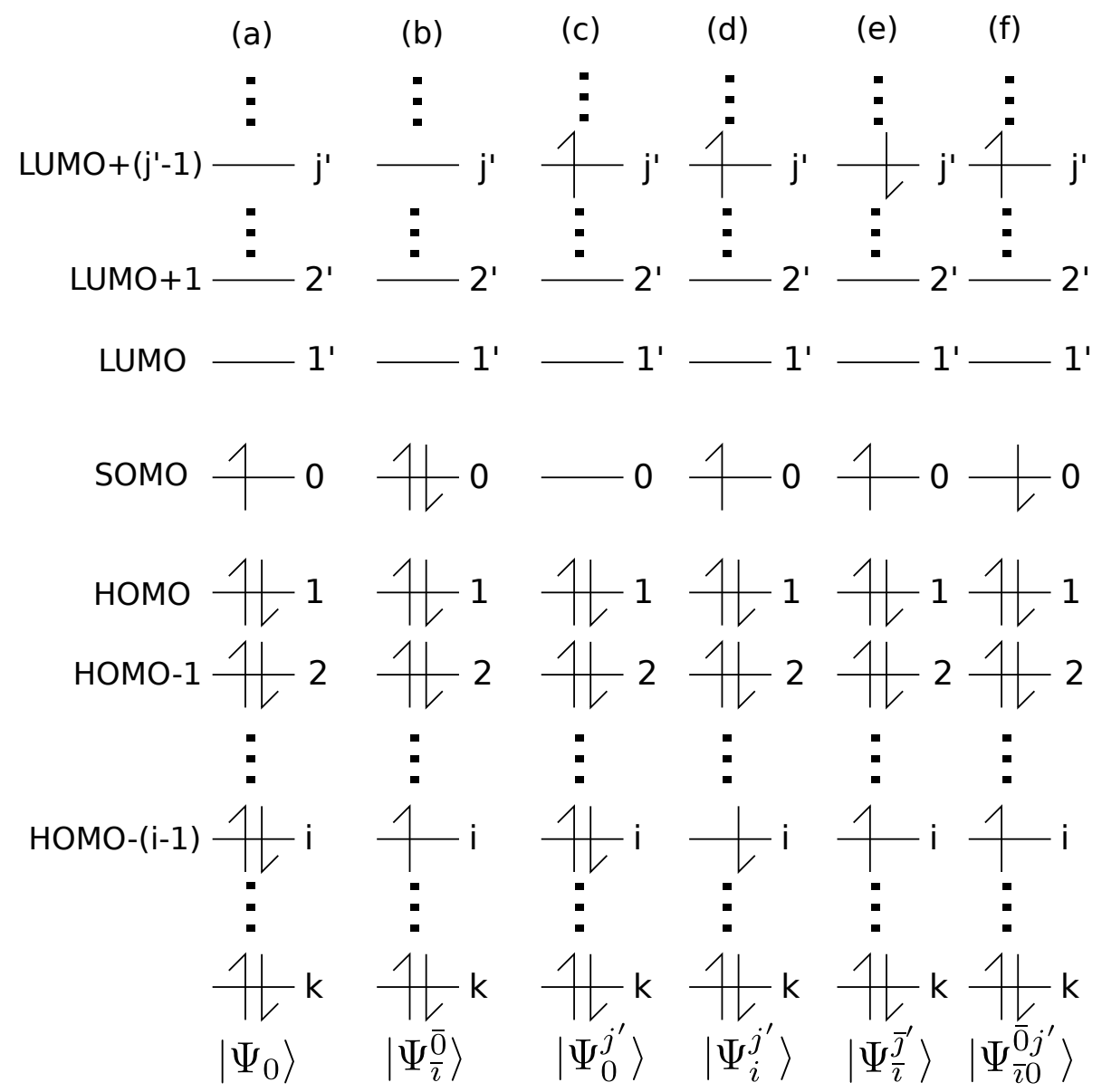

Figure 2. Molecular orbital diagrams showing various electronic states considered in the paper. On the left-hand side the orbitals are labelled with the usual labels (HOMO, SOMO etc) and the orbital numbering used in the article is used on the right-hand side of each diagram. Part (a) shows the ground state $\left|\Psi_{0}\right\rangle$, (b) is exciting a down-spin electron from (bonding) orbital $i$ to the SOMO, $\left|\Psi_{\bar{i}}^{\overline{0}}\right\rangle$, (c) to exciting the up-spin electron from the SOMO to (antibonding) orbital $j^{\prime}$, denoted $\left|\Psi_{0}^{j^{\prime}}\right\rangle$. Parts (d) and (e) show exciting an electron from bonding orbital $i$ to antibonding orbital $j^{\prime}$, (d) to exciting an up-spin electron $\left|\Psi_{i}^{j^{\prime}}\right\rangle$ and (e) to exciting a down-spin electron $\left|\Psi_{\bar{i}}^{\bar{j}^{\prime}}\right\rangle$. The in-phase combination of the excitations in (d) and (e) forms $\left|\Psi_{i}^{j^{\prime},+}\right\rangle$ as given in Eq. (4). Part (f) shows a double excitation from $\bar{\imath}$ to $\overline{0}$ and 0 to $j^{\prime}$, which has the same spatial orbital occupancy and $M_{s}=1 / 2$ value as (d) and (e) and which is taken in linear combinations with (d) and (e) to form spin-pure states.

In order for $\left|\Psi_{0}\right\rangle$ to be stationary (for our purposes, a minimum), $\delta E$ must be zero for all values of $\left\{\lambda_{i j}\right\}$, which is satisfied if and only if all Hamiltonian bra-kets in Eq. (5) are zero. These matrix elements are: ${ }^{22}$

$$
\begin{aligned}
\left\langle\Psi_{0}|\hat{H}| \Psi_{i}^{\overline{0}}\right\rangle & =h_{i 0}+\sum_{l=1}^{k}[2(i 0 \mid l l)-(i l \mid l 0)]+(i 0 \mid 00) \\
\left\langle\Psi_{0}|\hat{H}| \Psi_{0}^{j^{\prime}}\right\rangle & =h_{0 j^{\prime}}+\sum_{l=1}^{k}\left[2\left(0 j^{\prime} \mid l l\right)-\left(0 l \mid l j^{\prime}\right)\right] \\
\left\langle\Psi_{0}|\hat{H}| \Psi_{i}^{j^{\prime},+}\right\rangle & =\sqrt{2}\left\{h_{i j^{\prime}}+\sum_{l=1}^{k}\left[2\left(i j^{\prime} \mid l l\right)-\left(i l \mid l j^{\prime}\right)\right]+\frac{1}{2}\left[2\left(i j^{\prime} \mid 00\right)-\left(i 0 \mid 0 j^{\prime}\right)\right]\right\}
\end{aligned}
$$

where $h_{i j}$ corresponds to the one-electron integral $\left\langle\psi_{i}|\hat{h}(1)| \psi_{j}\right\rangle$ and $(i j \mid k l)$ is a two-electron spatial integral in 
the chemists' notation: ${ }^{26}$

$$
(i j \mid k l)=\int d \mathbf{r}_{1} \int d \mathbf{r}_{2} \psi_{i}^{*}\left(\mathbf{r}_{1}\right) \psi_{j}\left(\mathbf{r}_{1}\right) \frac{1}{r_{12}} \psi_{k}^{*}\left(\mathbf{r}_{2}\right) \psi_{l}\left(\mathbf{r}_{2}\right) .
$$

Careful examination of Eq. (6) suggests the following, approximate, Fock-like operator ${ }^{22}$

$$
\left\langle\psi_{p}|\hat{F}| \psi_{q}\right\rangle=F_{p q}=h_{p q}+\sum_{l=1}^{k}[2(p q \mid l l)-(p l \mid l q)]+\frac{1}{2}[2(p q \mid 00)-(p 0 \mid 0 q)]
$$

and by inserting Eq. (8) into Eq. (6) we obtain

$$
\begin{aligned}
F_{p 0}+\frac{1}{2}(p 0 \mid 00) & =0 \\
F_{0 q}-\frac{1}{2}(0 q \mid 00) & =0 \\
\sqrt{2} F_{p q} & =0
\end{aligned}
$$

where $p=1,2, \ldots, k$ and $q=1^{\prime}, 2^{\prime}, \ldots, \infty$ (we assume at present a theoretically infinite and complete basis set). Eq. (9) is not as straightforward an in the conventional closed-shell case, but by approximating that $(p 0 \mid 00)$ and $(0 q \mid 00)$ are small (and they can sometimes be shown to be zero by symmetry) Longuet-Higgins and Pople suggest simply considering ${ }^{22}$

$$
F_{p q}=0 .
$$

Strictly speaking, the equations in Eq. (9) only consider the case where $p$ is a doubly occupied orbital and $q$ is an empty orbital. However, since $\hat{F}$ is a linear and Hermitian operator, ${ }^{22}$ its eigenstates will have real eigenvalues and be orthogonal. Consequently the relevant molecular orbitals we require are simply eigenfunctions of $\hat{F}$

$$
\hat{F}\left|\psi_{l}\right\rangle=\epsilon_{l}\left|\psi_{l}\right\rangle,
$$

with eigenvalue, or orbital energy,

$$
\epsilon_{l}=F_{p p}=h_{p p}+\sum_{l=1}^{k}\left(2 J_{p l}-K_{p l}\right)+J_{p 0}-\frac{1}{2} K_{p 0},
$$

where we have used the standard definitions for the Coulomb and exchange integrals ${ }^{26}$

$$
\begin{aligned}
J_{p q} & =:(p p \mid q q), \\
K_{p q} & =:(p q \mid q p),
\end{aligned}
$$

and assumed, as in conventional electronic structure theory, that the orbitals are real.

Using standard electronic structure theory algebra ${ }^{26}$ the total electronic energy is

$$
\begin{aligned}
\left\langle\Psi_{0}|\hat{H}| \Psi_{0}\right\rangle & =\left(\sum_{l=1}^{k} 2 h_{l l}\right)+h_{00}+\sum_{l=1}^{k}\left(2 J_{0 l}-K_{0 l}+\sum_{m=1}^{k} 2 J_{l m}-K_{l m}\right) \\
& \equiv\left(\sum_{l=0}^{k} 2 h_{l l}\right)-h_{00}+\sum_{l=0}^{k} \sum_{m=0}^{k}\left(2 J_{l m}-K_{l m}\right)-\sum_{m=0}^{k}\left(2 J_{0 m}-K_{0 m}\right) \\
& =\sum_{l=1}^{k}\left(h_{l l}+F_{l l}\right)+\frac{1}{2}\left(h_{00}+F_{00}\right)-\frac{1}{4} J_{00} .
\end{aligned}
$$

Note that this is more complicated than for a conventional closed-shell system ${ }^{26}$ as there is only one electron in the SOMO. 


\section{EXCITED STATES}

We now consider the low-lying excited states of a radical. Longuet-Higgins and Pople ${ }^{22}$ only considered excitations involving the HOMO, SOMO and LUMO and here we consider the more general case where excitations involve any bonding orbital $i$ to any antibonding orbital $j^{\prime}$.

The singly excited state formed by exciting from $\bar{\imath}$ to the SOMO $\left(\left|\Psi_{\bar{\imath}}^{\overline{0}}\right\rangle\right.$, Fig. 2(b)), and from the SOMO to $j^{\prime}$

$\left(\left|\Psi_{0}^{j^{\prime}}\right\rangle\right.$, Fig. 2(c)) are considered first. Both these states are eigenstates of $\hat{S}_{z}$ (with eigenvalue $1 / 2$ ) and $\hat{S}^{2}$ (with eigenvalue $S(S+1)=3 / 4)$.

We then consider exciting from orbital $i$ to $j^{\prime}$, which is more complicated. From standard (non-relativistic) electronic structure theory, ${ }^{26}$ the electronic Hamiltonian commutes with $\hat{S}_{z}$ and $\hat{S}^{2}$, meaning that the eigenstates of of $\hat{H}$ must also be eigenstates of $\hat{S}_{z}$ and $\hat{S}^{2}$. The true (Full Configuration Interaction, FCI) eigenstates of a radical are generally too computationally expensive to determine, but (as mentioned earlier) it is useful to ensure that approximate eigenstates of $\hat{H}$ (such as those determined from considering a reduced space of orbital excitations) are also eigenstates of $\hat{S}_{z}$ and $\hat{S}^{2}$. When considering the action of $\hat{S}^{2}$ upon, for instance, $\left|\Psi_{i}^{j^{\prime}}\right\rangle$, a doubly excited state $\left|\Psi_{\bar{\imath} 0}^{\overline{0} j^{\prime}}\right\rangle$ is generated as part of the solution, corresponding to exciting an electron from $\bar{i}$ to $\overline{0}$ and then from 0 to $j^{\prime}$, as shown in Fig. 2(f). This excitation has the same spatial orbital occupancy as $\left|\Psi_{i}^{j^{\prime}}\right\rangle$ and $\left|\Psi_{\bar{\imath}}^{\bar{j}^{\prime}}\right\rangle$, and in order to obtain a complete set of spin-pure states with $M_{s}=+1 / 2$ and one electron in orbitals $i, 0$ and $j^{\prime}$, this double excitation must also be considered.

\subsection{Energies}

The energy of $\left|\Psi_{\bar{\imath}}^{\overline{0}}\right\rangle$ is

$$
\begin{aligned}
E\left(\Psi_{\bar{\imath}}^{\overline{0}}\right) & \equiv\left\langle\Psi_{\bar{\imath}}^{\overline{0}}|\hat{H}| \Psi_{\bar{\imath}}^{\overline{0}}\right\rangle \\
& =\left(\sum_{l=0}^{k} 2 h_{l l}\right)-h_{i i}+\sum_{l=0}^{k} \sum_{m=0}^{k}\left(2 J_{l m}-K_{l m}\right)-\sum_{l=0}^{k}\left(2 J_{i l}-K_{i l}\right) \\
& =E_{0}+F_{00}-F_{i i}-J_{i 0}+\frac{1}{2} K_{i 0}+\frac{1}{2} J_{00},
\end{aligned}
$$

and of $\left|\Psi_{0}^{j^{\prime}}\right\rangle$,

$$
\begin{aligned}
E\left(\Psi_{0}^{j^{\prime}}\right) & \equiv\left\langle\Psi_{0}^{j^{\prime}}|\hat{H}| \Psi_{0}^{j^{\prime}}\right\rangle \\
& =\left(\sum_{l=1}^{k} 2 h_{l l}\right)+h_{j^{\prime} j^{\prime}}+\sum_{l=1}^{k} \sum_{m=1}^{k}\left(2 J_{l m}-K_{l m}\right)+\sum_{l=1}^{k}\left(2 J_{l j^{\prime}}-K_{l j^{\prime}}\right) \\
& =E_{0}+F_{j^{\prime} j^{\prime}}-F_{00}-J_{0 j^{\prime}}+\frac{1}{2} K_{0 j^{\prime}}+\frac{1}{2} J_{00} .
\end{aligned}
$$

As discussed above, evaluation of states with one electron in orbitals $i, 0$ and $j^{\prime}$ is more complicated. We firstly find the Hamiltonian matrix in the basis of $\left\{\left|\Psi_{i}^{j^{\prime}}\right\rangle,\left|\Psi_{\bar{\imath}}^{\bar{j}^{\prime}}\right\rangle,\left|\Psi_{\bar{\imath} 0}^{\overline{0} j^{\prime}}\right\rangle\right\}$ (Fig. 2(d), (e) and (f) respectively)

$$
\begin{aligned}
\mathbf{H}= & {\left[\left(\sum_{l=1}^{k} 2 h_{l l}\right)+h_{00}-h_{i i}+h_{j^{\prime} j^{\prime}}+\sum_{l=1}^{k} \sum_{m=1}^{k}\left(2 J_{l m}-K_{l m}\right)\right.} \\
& \left.+\sum_{l=1}^{k}\left(-2 J_{i l}+K_{i l}+2 J_{0 l}-K_{0 l}+2 J_{l j^{\prime}}-K_{l j^{\prime}}\right)-J_{i 0}-J_{i j^{\prime}}+J_{0 j^{\prime}}+K_{i 0}+K_{i j^{\prime}}\right] \mathbf{I} \\
& +\left(\begin{array}{lll}
-K_{0 j^{\prime}} & +K_{i j^{\prime}} & -K_{i 0} \\
+K_{i j^{\prime}} & -K_{i 0} & +K_{0 j^{\prime}} \\
-K_{i 0} & +K_{0 j^{\prime}} & -K_{i j^{\prime}}
\end{array}\right)
\end{aligned}
$$


with associated spin Hamiltonian matrix

$$
\mathbf{S}^{2}=\left(\begin{array}{ccc}
7 / 4 & -1 & +1 \\
-1 & +7 / 4 & -1 \\
+1 & -1 & +7 / 4
\end{array}\right)
$$

This matrix has eigenvalues of $3 / 4=(1 / 2) \times(1 / 2+1)$ twice, corresponding to two doublet states, and $15 / 4=$ $(3 / 2) \times(3 / 2+1)$, corresponding to one quartet state. The quartet eigenstate is

$$
\left|{ }^{4} \Psi_{i}^{j^{\prime}}\right\rangle=\frac{1}{\sqrt{3}}\left(\left|\Psi_{i}^{j^{\prime}}\right\rangle-\left|\Psi_{\bar{\imath}}^{\bar{\jmath}^{\prime}}\right\rangle+\left|\Psi_{\bar{\imath} 0}^{\overline{0} j^{\prime}}\right\rangle\right) \mapsto \frac{1}{\sqrt{3}}\left(\begin{array}{c}
1 \\
-1 \\
1
\end{array}\right)
$$

and the doublet eigenstates can be chosen to be $\left|\Psi_{i}^{j^{\prime},+}\right\rangle$ considered earlier

$$
\left|\Psi_{i}^{j^{\prime},+}\right\rangle=\frac{1}{\sqrt{2}}\left(\left|\Psi_{i}^{j^{\prime}}\right\rangle+\left|\Psi_{i}^{\bar{j}^{\prime}}\right\rangle\right) \mapsto \frac{1}{\sqrt{2}}\left(\begin{array}{l}
1 \\
1 \\
0
\end{array}\right)
$$

and a second doublet state which we define as

$$
\left|\Psi_{i}^{j^{\prime},-}\right\rangle=: \frac{1}{\sqrt{6}}\left(-\left|\Psi_{i}^{j^{\prime}}\right\rangle+\left|\Psi_{\bar{\imath}}^{\bar{j}^{\prime}}\right\rangle+2\left|\Psi_{\bar{\imath} 0}^{\overline{0} j^{\prime}}\right\rangle\right) \mapsto \frac{1}{\sqrt{6}}\left(\begin{array}{c}
-1 \\
1 \\
2
\end{array}\right)
$$

where ' $\mapsto$ ' means maps to the vector representation of $\left\{\left|\Psi_{i}^{j^{\prime}}\right\rangle,\left|\Psi_{\bar{\imath}}^{\bar{\jmath}^{\prime}}\right\rangle,\left|\Psi_{\bar{\imath} 0}^{\overline{0} j^{\prime}}\right\rangle\right\}$. Rotating the Hamiltonian in Eq. (18) to the basis of $\left\{\left|{ }^{4} \Psi_{i}^{j^{\prime}}\right\rangle,\left|\Psi_{i}^{j^{\prime},+}\right\rangle,\left|\Psi_{i}^{j^{\prime},-}\right\rangle\right\}$ we obtain

$$
\begin{aligned}
\mathbf{H}= & {\left[E_{0}-F_{i i}+F_{j^{\prime} j^{\prime}}-J_{i j^{\prime}}+\frac{1}{2} K_{i 0}+\frac{1}{2} K_{0 j^{\prime}}+K_{i j^{\prime}}\right] \mathbf{I} } \\
& +\left(\begin{array}{ccc}
-\left(K_{0 j^{\prime}}+K_{i j^{\prime}}+K_{i 0}\right) & 0 & 0 \\
0 & K_{i j^{\prime}}-\frac{1}{2}\left(K_{0 j^{\prime}}+K_{i 0}\right) & \frac{\sqrt{3}}{2}\left(K_{0 j^{\prime}}-K_{i 0}\right) \\
0 & \frac{\sqrt{3}}{2}\left(K_{0 j^{\prime}}-K_{i 0}\right) & -K_{i j^{\prime}}+\frac{1}{2}\left(K_{0 j^{\prime}}+K_{i 0}\right)
\end{array}\right),
\end{aligned}
$$

from which we find

$$
\begin{aligned}
E\left({ }^{4} \Psi_{i}^{j^{\prime}}\right) & =E_{0}-F_{i i}+F_{j^{\prime} j^{\prime}}-J_{i j^{\prime}}-\frac{1}{2} K_{i 0}-\frac{1}{2} K_{0 j^{\prime}}, \\
E\left(\Psi_{i}^{j^{\prime},+}\right) & =E_{0}-F_{i i}+F_{j^{\prime} j^{\prime}}-J_{i j^{\prime}}+2 K_{i j^{\prime}}, \\
E\left(\Psi_{i}^{j^{\prime},-}\right) & =E_{0}-F_{i i}+F_{j^{\prime} j^{\prime}}-J_{i j^{\prime}}+K_{i 0}+K_{0 j^{\prime}} .
\end{aligned}
$$

All states have as a component of their energy expression the energy of the ground state $E_{0}$, minus the energy of the orbital the electron is excited from, plus the energy or the orbital the electron is excited to, less the Coulomb attraction between an electron in $j^{\prime}$ and a hole in $i$. Furthermore, the energy of $\left|\Psi_{i}^{j^{\prime},{ }^{+}}\right\rangle$has the same functional form as the standard expression for a singlet exciton in a closed shell molecule ${ }^{26}{ }^{2}$ viz. the ground-state energy plus the energy of the orbtial the electron is excited to, minus the energy of the orbital the electron is excited from, minus the Coulomb attraction of the electron and hole, plus an exchange 'penalty' of $2 K_{i j^{\prime}}$. Since exchange integrals are always positive or zero, ${ }^{32}$ the quartet state is lower in energy than the doublets, as expected from Hund's rules.

These results are more general than those in Ref. 22 which only considers excitations within the HOMO, SOMO and LUMO manifold (equivalent to setting $i=1$ and $j=1$ in the notation used here). Note that $\left\langle\Psi_{i}^{j^{\prime},+}|\hat{H}| \Psi_{i}^{j^{\prime},-}\right\rangle \neq 0$ and in general these two states will be mixed by the Hamiltonian. ${ }^{\dagger}$

${ }^{\dagger}$ These states are eigenstates of $\hat{S}_{z}$ and $\hat{S}^{2}$ with the same eigenvalues of $1 / 2$ and $3 / 4$ respectively, such that there is no quantum mechanical reason why they should not mix. 


\subsection{Transition dipole moment}

Using standard electronic structure theory algebra ${ }^{26}$ we find

$$
\begin{aligned}
\left\langle\Psi_{0}|\hat{\mu}| \Psi_{\bar{\imath}}^{\overline{0}}\right\rangle & =\langle i|\hat{\mu}| 0\rangle, \\
\left\langle\Psi_{0}|\hat{\mu}| \Psi_{0}^{j^{\prime}}\right\rangle & =\left\langle 0|\hat{\mu}| j^{\prime}\right\rangle, \\
\left\langle\Psi_{0}|\hat{\mu}| \Psi_{i}^{j^{\prime}}\right\rangle & =\left\langle i|\hat{\mu}| j^{\prime}\right\rangle, \\
\left\langle\Psi_{0}|\hat{\mu}| \Psi_{\bar{\imath}}^{\bar{j}^{\prime}}\right\rangle & =\left\langle i|\hat{\mu}| j^{\prime}\right\rangle, \\
\left\langle\Psi_{0}|\hat{\mu}| \Psi_{\overline{0}}^{0} j^{\prime}\right\rangle & =0, \\
\left\langle\Psi_{0}|\hat{\mu}| \Psi_{i}^{j^{\prime},+}\right\rangle & =\sqrt{2}\left\langle i|\hat{\mu}| j^{\prime}\right\rangle .
\end{aligned}
$$

In addition the standard expression for the permanent dipole moment of a molecule in our notation is ${ }^{26}$

$$
\vec{\mu}=\left\langle\Psi_{0}\left|-\sum_{i=1}^{k} \mathbf{r}_{i}\right| \Psi_{0}\right\rangle+\sum_{A} Z_{A} \mathbf{r}_{A} .
$$

We find later that these expressions simplify significantly for alternant hydrocarbons.

\section{ATOMIC ORBITAL BASIS}

To convert the eigenvalue equation in Eq. (11) into a form which is amenable to computation we use the standard Linear Combination of Atomic Orbitals (LCAO) approximation, defining ${ }^{22}$

$$
\left|\psi_{p}\right\rangle=\sum_{\nu} C_{\nu p}\left|\phi_{\nu}\right\rangle
$$

where the summation extends over all atomic orbitals $\left\{\left|\phi_{\nu}\right\rangle\right\}$ with orbital coefficient $C_{\nu p}$. In accordance with convention $^{22}$ we use Greek letters for atomic orbitals and Roman letters for molecular orbitals and we omit the $\mathbf{r}$ dependence of orbitals for simplicity.

Inserting Eq. (27) into Eq. (11) we find

$$
\sum_{\nu} C_{\nu p} \hat{F}\left|\phi_{\nu}\right\rangle=\epsilon_{p} \sum_{\nu} C_{\nu p}\left|\phi_{\nu}\right\rangle
$$

multiplying on the right by $\left\langle\phi_{\mu}\right|$ and integrating over all space leads to

$$
\sum_{\nu}\left\langle\phi_{\mu}|\hat{F}| \phi_{\nu}\right\rangle C_{\nu p}=\sum_{\nu}\left\langle\phi_{\mu} \mid \phi_{\nu}\right\rangle C_{\nu p} \epsilon_{p}
$$

which can be more succinctly written as

$$
\mathbf{F C}=\mathrm{SCE}
$$

where we have defined the atomic orbital Fock matrix terms and overlap integrals as well as an orbital energy matrix:

$$
\begin{aligned}
F_{\mu \nu} & =\left\langle\phi_{\mu}|\hat{F}| \phi_{\nu}\right\rangle, \\
S_{\mu \nu} & =\left\langle\phi_{\mu} \mid \phi_{\nu}\right\rangle, \\
E_{p q} & =\epsilon_{p} \delta_{p q} .
\end{aligned}
$$

Equation (30) is similar to, but not exactly the same as, the conventional Roothaan equations [cf. Equation (3.139) of Ref 26], since the Fock operator for the radical in Eq. (8) is not the same as the conventional Fock operator [cf. Equation (3.154) of Ref 26]. 
To find an expression for the Fock matrix in the atomic orbital basis, we combine Eq. (8) and Eq. (27), giving

$$
F_{\mu \nu}=h_{\mu \nu}+\sum_{\rho} \sum_{\sigma}\left\{[2(\mu \nu \mid \rho \sigma)-(\mu \sigma \mid \rho \nu)]\left(\sum_{l=1}^{k} C_{\rho l}^{*} C_{\sigma l}\right)+\frac{1}{2}[2(\mu \nu \mid \rho \sigma)-(\mu \sigma \mid \rho \nu)] C_{\rho 0}^{*} C_{\sigma 0}\right\},
$$

which suggests defining a density matrix

$$
P_{\rho \sigma}=\left(\sum_{l=1}^{k} 2 C_{\rho l}^{*} C_{\sigma l}\right)+C_{\rho 0}^{*} C_{\sigma 0}
$$

such that

$$
F_{\mu \nu}=h_{\mu \nu}+\sum_{\rho} \sum_{\sigma} P_{\rho \sigma}\left[(\mu \nu \mid \rho \sigma)-\frac{1}{2}(\mu \sigma \mid \rho \nu)\right] .
$$

This equation, coupled with Eq. (30), therefore form the basis for radical electronic structure calculation.

Thus far, our equations have been completely general for monoradicals; that is, they does not require presumption of zero differential overlap or considering the $\pi$ system to be separate from the $\sigma$ system. While this is advantageous for high-level computation, in general it is difficult to derive general rules and guiding principles without making certain simplifications.

\section{APPLICATION OF PPP APPROXIMATIONS}

We now consider approximating the self-consistent equations of the previous sections in order to derive general predictive rules for radical chromophores. The approximations Longuet-Higgins and Pople use ${ }^{22}$ are essentially those of conventional Pariser-Parr-Pople theory, ${ }^{12-14}$ namely

1. The $\sigma$-system is treated as a non-polarizable core and its interaction with the $\pi$ system is accounted for in $\left\{h_{\mu \nu}\right\}$, which is treated as an empirical parameter

2. The neglect of differential overlap (NDO) approximation, which assumes that $\left\langle\phi_{\mu} \mid \phi_{\nu}\right\rangle=\delta_{\mu \nu}$, where $\delta_{\mu \nu}$ is the Kroenecker delta. This is equivalent to the overlap matrix being the identity matrix, viz. $\mathbf{S}=\mathbf{I}$.

3. Two electron integrals which depend on the overlap of two $p$ orbitals on different atoms are neglected, i.e.

$$
\begin{aligned}
(\mu \nu \mid \rho \sigma) & \simeq(\mu \mu \mid \rho \rho) \delta_{\mu \nu} \delta_{\rho \sigma} \\
& =\gamma_{\mu \rho} \delta_{\mu \nu} \delta_{\rho \sigma}
\end{aligned}
$$

where $\gamma_{\mu \rho}$ is a function which depends on the distance between atoms $\mu$ and $\rho$, i.e. $\gamma_{\mu \rho} \equiv \gamma_{\mu \rho}\left(\left|\mathbf{r}_{\mu}-\mathbf{r}_{\rho}\right|\right)$. Various parameterizations ${ }^{16,31}$ exist for this function, such that at large $\left|\mathbf{r}_{\mu}-\mathbf{r}_{\rho}\right|$ it has Coulombic $\left(|\mathbf{r}|^{-1}\right)$ dependence and at $|\mathbf{r}|=0$ is equal to a Hubbard-like repulsion term.

4. The attraction between an electron on atom $\mu$ and the nucleus of atom $\nu$ is approximated to be

$$
\left\langle\phi_{\mu}\left|\frac{-Z_{\nu}^{\mathrm{eff}}}{\left|r_{\mu}-r_{\nu}\right|}\right| \phi_{\mu}\right\rangle \simeq-Z_{\nu}^{\mathrm{eff}} \gamma_{\mu \nu}
$$

where $Z_{\alpha}^{\text {eff }}$ is the effective nuclear charge of atom $\alpha$ (after accounting for the $\sigma$ system). For carbon atoms this is simply $Z_{\alpha}^{\text {eff }}=1$.

Combining these approximations we set

$$
h_{\mu \mu}=\alpha_{\mu}-\sum_{\mu \neq \nu} Z_{\nu}^{\mathrm{eff}} \gamma_{\mu \nu}
$$


where $\alpha_{\mu}$ is the on-site energy (Hückel $\alpha$ ) term, and

$$
h_{\mu \nu}=\beta_{\mu \nu}, \quad \mu \neq \nu
$$

where $\beta_{\mu \nu}$ is the Hückel $\beta$ term, usually taken to be a constant value if the two atoms are adjacent and zero otherwise. However, in what follows the $\beta_{\mu \nu}$ value can vary, provided that it is non-zero only between adjacent atoms. Note that the interaction between an electron on atom $\mu$ and the charge on atom $\mu$ is included implicitly in $\alpha_{\mu}$.

Combining these approximations with the formula for the Fock matrix in Eq. (34) we find ${ }^{22}$

$$
\begin{aligned}
& F_{\mu \mu}=\alpha_{\mu}+\frac{1}{2} P_{\mu \mu} \gamma_{\mu \mu}+\sum_{\nu \neq \mu} \gamma_{\mu \nu}\left(P_{\nu \nu}-Z_{\nu}^{\mathrm{eff}}\right) \\
& F_{\mu \nu}=\beta_{\mu \nu}-\frac{1}{2} P_{\nu \mu} \gamma_{\mu \nu}, \quad \mu \neq \nu
\end{aligned}
$$

In the next section we will see that these expressions simplify further for the case of alternant hydrocarbon radicals.

\section{ALTERNANT RADICAL HYDROCARBONS}

We now consider the specific case of radicals which are alternant hydrocarbons, ${ }^{20,22,25}$ that is, only carbon atoms are in the conjugated system, and the carbon atoms can be divided into two classes, denoted starred and unstarred, such that no two atoms from the same class are adjacent. Radicals such as the allyl radical, benzyl radical and triphenylmethyl radical are alternant, whereas the cyclopentadienyl radical is not.

As we are considering monoradicals with an odd number of electrons, there will be $R=(N+1) / 2$ carbon atoms in one class, which we denote the starred class $(\star)$ and $R-1=(N-1) / 2$ carbon atoms which are unstarred (o). There may be situations in which the starred and unstarred atoms are more unevenly distributed that this, but we do not consider such cases here. We can, without loss of generality, list all the starred atoms first in the atomic orbital basis, then all the unstarred atoms.

We firstly consider the the solution to the Fock matrix for an alternant hydrocarbon where we set the density matrix $\mathbf{P}=\mathbf{0}$, such that the Fock matrix reduces to a Hückel Hamiltonian. Due to the grouping of starred then unstarred carbon atoms this matrix has a block form:

$$
\mathbf{F}(\mathbf{P}=\mathbf{0})=\left(\begin{array}{cc}
\alpha \mathbf{I}_{R} & \mathbf{B} \\
\mathbf{B}^{T} & \alpha \mathbf{I}_{R-1}
\end{array}\right)
$$

where $\mathbf{I}_{R}$ is the $R \times R$ identity matrix, and $\mathbf{B}$ is the a rectangular matrix of $R$ rows and $R-1$ columns.

In general, the Fock matrix is $N \times N$, so will have $N$ eigenvalues and distinct eigenvectors. Some of the eigenvalues may be zero or degenerate, as we shall see.

We now consider an arbitrary eigenvalue to $\mathbf{F}(\mathbf{P}=\mathbf{0})$ where we denote the first $R$ coefficients as $\mathbf{c}^{\star}$ and the remaining $R-1$ as $\mathbf{c}^{\circ}$. As it is an eigenvalue we can by construction write

$$
\left(\begin{array}{cc}
\alpha \mathbf{I}_{R} & \mathbf{B} \\
\mathbf{B}^{T} & \alpha \mathbf{I}_{R-1}
\end{array}\right)\left(\begin{array}{l}
\mathbf{c}^{\star} \\
\mathbf{c}^{\circ}
\end{array}\right)=\left(\begin{array}{c}
\alpha \mathbf{c}^{\star}+\mathbf{B} \mathbf{c}^{\circ} \\
\mathbf{B}^{T} \mathbf{c}^{\star}+\alpha \mathbf{c}^{\circ}
\end{array}\right)=\lambda\left(\begin{array}{l}
\mathbf{c}^{\star} \\
\mathbf{c}^{\circ}
\end{array}\right)
$$

Let us now consider a vector similar to the eigenvector in the previous equation, but with the sign of all unstarred coefficients reversed,

$$
\left(\begin{array}{cc}
\alpha \mathbf{I}_{R} & \mathbf{B} \\
\mathbf{B}^{T} & \alpha \mathbf{I}_{R-1}
\end{array}\right)\left(\begin{array}{c}
\mathbf{c}^{\star} \\
-\mathbf{c}^{\circ}
\end{array}\right)=\left(\begin{array}{c}
\alpha \mathbf{c}^{\star}-\mathbf{B} \mathbf{c}^{\circ} \\
-\mathbf{B}^{T} \mathbf{c}^{\star}+\alpha \mathbf{c}^{\circ}
\end{array}\right)=(2 \alpha-\lambda)\left(\begin{array}{c}
\mathbf{c}^{\star} \\
\mathbf{c}^{\circ}
\end{array}\right)
$$

and we see that this is also an eigenvector of $\mathbf{F}(\mathbf{P}=\mathbf{0})$ with eigenvalue $(2 \alpha-\lambda)$. This means that the eigenvectors occur in pairs of energy $\lambda$ and $2 \alpha-\lambda$. However, there are an odd number of orbitals, and therefore an odd number of eigenvalues. The only way to reconcile this with eigenvalues occuring in pairs is if one eigenvalue is its own pair, i.e. $\lambda=2 \alpha-\lambda$. 
Inserting this condition into Eq. (41) gives

$$
\begin{aligned}
\mathbf{B c}^{\circ} & =\mathbf{0}, \\
\mathbf{B}^{T} \mathbf{c}^{\star} & =\mathbf{0}
\end{aligned}
$$

Eq. (43a) has $R-1$ unknowns (scalar variables in $\mathbf{c}^{\circ}$ ) and $R$ conditions (the length of the $\mathbf{0}$ vector) and in general the only solution to Eq. (43a) is $\mathbf{c}^{\circ}=\mathbf{0}$. However, Eq. (43b) has $R$ variables and $R-1$ conditions, and in general it will always be possible to find a nontrivial solution for $\mathbf{c}^{\star} \neq \mathbf{0}$. By the pairing properties discussed above, the solution $\lambda=\alpha$ will have $R-1$ solutions of lower energy and $R-1$ solutions of higher energy, and, further to the orbital numbering scheme above, this solution is therefore corresponds to $\epsilon_{0}$, the SOMO.

We now consider the properties of the solution obtained from the Hückel-like equations. By combining the pairing properties of the eigenvectors with the definition of the density matrix in Eq. (33) we see that, if $\rho$ and $\sigma$ are both starred, or both unstarred,

$$
P_{\rho \sigma}=\sum_{l=1}^{k}\left(C_{\rho l}^{*} C_{\sigma l}+C_{\rho l^{\prime}}^{*} C_{\sigma l^{\prime}}\right)+C_{\rho 0} C_{\sigma 0}=\delta_{\rho \sigma}
$$

where we have used the orthogonality of the eigenvectors of a Hermitian (or real symmetric) matrix.

Inserting Eq. (44) into Eq. (39) and setting $Z_{\nu}^{\text {eff }}=1$ for a carbon atom,

$$
\begin{aligned}
& F_{\mu \mu}=\alpha_{\mu}+\frac{1}{2} \gamma_{\mu \mu} \\
& F_{\mu \nu}=0 \text { Both atoms starred or both unstarred } \\
& F_{\mu \nu}=\beta_{\mu \nu}-\frac{1}{2} P_{\nu \mu} \gamma_{\mu \nu} \text { Otherwise }
\end{aligned}
$$

We now see that, including a non-zero density matrix, the Fock matrix has the form

$$
\mathbf{F}(\mathbf{P})=\left(\begin{array}{cc}
\left(\alpha+\frac{1}{2} \gamma_{\mu \mu}\right) \mathbf{I}_{R} & \mathbf{B}(\mathbf{P}) \\
\mathbf{B}(\mathbf{P})^{T} & \left(\alpha+\frac{1}{2} \gamma_{\mu \mu}\right) \mathbf{I}_{R-1}
\end{array}\right)
$$

which is very similar to that in Eq. (40). By using similar reasoning to the above, it follows that the eigenvalues of $\mathbf{F}(\mathbf{P})$ will be paired such that for eigenvalue $\lambda_{i}$ with eigenvector $\left(\begin{array}{l}\mathbf{c}^{\star} \\ \mathbf{c}^{\circ}\end{array}\right)$, there exists an eigenvector $\left(\begin{array}{c}\mathbf{c}^{\star} \\ -\mathbf{c}^{\circ}\end{array}\right)$ with eigenvalue $\lambda_{i^{\prime}}=2 \alpha+\gamma_{\mu \mu}-\lambda_{i}$. The SOMO is at energy $\lambda_{0}=\alpha+\gamma_{\mu \mu} / 2$ with no amplitude on any of the $R-1$ unstarred carbons and amplitude only on the $R$ starred carbon atoms. It therefore follows by induction that, as the first iteration of the self-consistent field procedure has alternacy symmetry, and for the $N$ th iteration, the $(N+1)$ th iteration will also have alternacy symmetry, so will the eventual solution.

We can consequently state for the converged molecular orbitals: ${ }^{22}$

1. For a doubly occupied, bonding orbital $l$, with coefficients $C_{\rho l}$ and energy $\epsilon_{l} \equiv F_{l l}$, there exists a corresponding empty, antibonding orbital $l^{\prime}$ with energy

$$
F_{l^{\prime} l^{\prime}}=2 \alpha+\gamma_{\mu \mu}-F_{l l}
$$

and coefficients

$$
C_{\rho l^{\prime}}= \begin{cases}C_{\rho l} & \rho \text { starred } \\ -C_{\rho l} & \rho \text { unstarred }\end{cases}
$$

It immediately follows that

$$
C_{\rho l}^{*} C_{\rho m}=C_{\rho l^{\prime}}^{*} C_{\rho m^{\prime}}
$$

whether or not atom $\rho$ is starred or unstarred. 
2. The SOMO is located only on starred atoms such that

$$
C_{\rho 0}=0, \quad \rho \text { unstarred, }
$$

and has energy

$$
F_{00}=\alpha+\frac{1}{2} \gamma_{\mu \mu} .
$$

3. As a result of Eq. (47) and Eq. (51),

$$
\begin{aligned}
\epsilon_{l^{\prime}}-\epsilon_{0} & =\epsilon_{0}-\epsilon_{l} \\
\epsilon_{l^{\prime}}-\epsilon_{m} & =\epsilon_{m^{\prime}}-\epsilon_{l}
\end{aligned}
$$

such that the orbital energies are symmetrically distributed around the SOMO.

4. For a two electron integral in the molecular orbital basis, by combining the orbital pairing properties in Eq. (48) and Eq. (50) with the definition of the atomic orbital basis in Eq. (27) and the neglect of differential overal in Eq. (35) we see

$$
\begin{aligned}
(l m \mid p q) & =\sum_{\mu} \sum_{\rho} C_{\mu l}^{*} C_{\mu m} C_{\rho p}^{*} C_{\rho q} \gamma_{\mu \rho} \\
& =\left(l^{\prime} m^{\prime} \mid p q\right)=\left(l m \mid p^{\prime} q^{\prime}\right)=\left(l^{\prime} m^{\prime} \mid p^{\prime} q^{\prime}\right), \\
(l 0 \mid p q) & =\left(l^{\prime} 0 \mid p q\right) .
\end{aligned}
$$

It immediately follows from the definition of the Coulomb and Exchange integrals ${ }^{26}$ that

$$
\begin{aligned}
J_{l m} & =J_{l^{\prime} m}=J_{l m^{\prime}}=J_{l^{\prime} m^{\prime}}, \\
J_{0 j} & =J_{0 j^{\prime}}, \\
K_{l m} & =\left(l^{\prime} m^{\prime} \mid l m\right)=\left(l m \mid l^{\prime} m^{\prime}\right)=K_{l^{\prime} m^{\prime}}, \\
K_{l^{\prime} m} & =K_{l m^{\prime}}, \\
K_{l 0} & =K_{l^{\prime} 0} .
\end{aligned}
$$

5. For the dipole moment between spatial orbitals $l$ and $m$, denoted, $\left\langle\psi_{l}|\hat{\mu}| \psi_{m}\right\rangle$, with the neglect of differential overlap this becomes ${ }^{13,21}$

$$
\left\langle\psi_{l}|\hat{\mu}| \psi_{m}\right\rangle \equiv\langle l|\hat{\mu}| m\rangle=-e \sum_{\nu} C_{\nu l}^{*} \mathbf{r}_{\nu} C_{\nu m}
$$

Combining this result with the alternacy properties we see

$$
\begin{aligned}
\langle l|\hat{\mu}| m\rangle & =\left\langle l^{\prime}|\hat{\mu}| m^{\prime}\right\rangle \\
\langle l|\hat{\mu}| 0\rangle & =\left\langle l^{\prime}|\hat{\mu}| 0\right\rangle
\end{aligned}
$$

6. For excited states we see

$$
\begin{aligned}
E\left(\Psi_{\bar{\imath}}^{\overline{0}}\right) & =E\left(\Psi_{0}^{i^{\prime}}\right) \\
\left\langle\Psi_{\bar{\imath}}^{\overline{0}}|\hat{H}| \Psi_{0}^{i^{\prime}}\right\rangle & =K_{i 0} .
\end{aligned}
$$

This means that the $i \rightarrow 0$ and $0 \rightarrow i^{\prime}$ excitations are degenerate, even including two-electron effects. We can therefore define

$$
\left|\Psi_{i 0}^{ \pm}\right\rangle=\frac{1}{\sqrt{2}}\left(\left|\Psi_{\bar{\imath}}^{\overline{0}}\right\rangle \pm\left|\Psi_{0}^{i^{\prime}}\right\rangle\right)
$$


with energies

$$
E\left(\Psi_{i 0}^{ \pm}\right)=E_{0}+F_{00}-F_{i i}-J_{i 0}+\frac{1}{2} K_{i 0}+\frac{1}{2} J_{00} \pm K_{i 0}
$$

In this notation, the minus combination is of lower energy. Note that if the electronic states are defined with the orbitals in a different order (as in Ref. 21 and adopted in Ref. 22) then antisymmetry properties may lead to the plus combination being of lower energy. The choice of orbital notation, of, course, does not affect the prediction of any observable property such as dipole moment.

7. For the transition dipole moment of $\left|\Psi_{i 0}^{ \pm}\right\rangle$excited states, we find, in our notation,

$$
\begin{aligned}
\left\langle\Psi_{0}|\hat{\mu}| \Psi_{i 0}^{-}\right\rangle & =0 \\
\left\langle\Psi_{0}|\hat{\mu}| \Psi_{i 0}^{+}\right\rangle & =\sqrt{2}\langle i|\hat{\mu}| 0\rangle
\end{aligned}
$$

where we have used the alternacy properties and assumed, as in conventional electronic structure theory, that the orbitals are real.

The 'plus' and 'minus' states $\left|\Psi_{i 0}^{ \pm}\right\rangle$are somewhat similar to pseudoparity in Pariser's interpretation of PPP theory, ${ }^{13}$ but here the dark state is always of lower energy (from Eq. (59) and since exchange integrals are always positive $\mathrm{e}^{13,32}$ ), whereas this is not necessarily the case with plus and minus states in closed-shell alternant hydrocarbons. ${ }^{13}$

8. When exciting orbital $i$ to $j^{\prime}$,

$$
E\left(\Psi_{i}^{j^{\prime},+}\right)=E\left(\Psi_{j}^{i^{\prime},+}\right)
$$

However, the mixing of these states is significantly more complex than for the case of $\Psi_{i 0}^{ \pm}$, and as far as we are aware was not considered by Longuet-Higgins and Pople for $i \neq j .^{22}$ A full analysis of this is left as further work.

9. For the permanent dipole moment of the ground state $\left|\Psi_{0}\right\rangle$, using Eq. (26) and treating the nuclei as having effective nuclear charge $Z_{\nu}^{\text {eff }}$ we find

$$
\vec{\mu}=-\sum_{\nu}\left(P_{\nu \nu}-Z_{\nu}^{\mathrm{eff}}\right) \mathbf{r}_{\nu}
$$

For an alternant hydrocarbon as $P_{\nu \nu}=Z_{\nu}^{\text {eff }}=1$ it immediately follows that

$$
\vec{\mu}=\mathbf{0},
$$

such that the ground-state molecule will have no dipole moment. It is straightforward to generalise this to show that an alternant hydrocarbon radical will have no higher multipole (quadrupole etc) either.

The results in 1-3 are those found by Longuet-Higgins and Pople. The results in 4-9 are similar to those found by Pariser for closed-shell molecules ${ }^{13}$ and some of the results by Dewar, Longuet-Higgins and Pople are special cases of them ${ }^{21,22}$ but we believe these general results for radicals have not appeared in the literature before and are the central results of this paper.

\section{APPLICATION TO ORGANIC LIGHT-EMITTING DIODES}

In this section we consider how the theoretical results derived above can help inform the design and properties of organic light-emitting diodes and other optoelectronics. Unless otherwise stated, these results are for alternant hydrocarbon radicals.

Organic radicals commmonly used in OLEDs such as TTM and PTM are chlorinated derivatives of alternant radicals. ${ }^{11}$ Strictly speaking, the chlorine atoms may interact with the hydrocarbon $\pi$ system in these molecules, but to a good approximation this interaction can be neglected since the large size of chlorine $3 p$ orbitals compared to carbon $2 \mathrm{p}$, and their much higher electronegativity, is likely to make any such interaction minimal. ${ }^{1}$ This means that we can reasonably expect molecules such as TTM and PTM to display the same properties, discussed below, as a conventional alternant hydrocarbon radical. 


\subsection{Alternacy symmetry and the SOMO}

From standard frontier molecular orbital theory, the radical is likely to react via the SOMO. The SOMO of an alternant hydrocarbon radical is found only on the $(N+1) / 2$ starred atoms. In general, the radical molecule reacting chemically is undesirable in an optoelectronic device, and it is therefore advisable to protect atoms through which the radical might react by bonding substituents to them (such that the radical is sterically hindered). The question then arises as to which atoms in particular in the conjugated system require protection, and these will be the $(N+1) / 2$ starred atoms. It is no surprise, therefore, that it is these atoms which are protected by chlorines in the TTM (tris-2,4,6-trichlorophenylmethyl) radical. ${ }^{3,11}$ We therefore advise that in the design of future radical emitters based on a hydrocarbon structure the SOMO atoms (which can be deduced without computation) are protected.

\subsection{Absorption and emission of radicals}

Taken together, the results in section 7 mean that, in an alternant hydrocarbon radical, the low-energy region of the spectrum will show an extremely weak $D_{1}$ absorption, corresponding to $\left|\Psi_{i 0}^{-}\right\rangle$, and an intense, high-energy absorption corresponding to $\left|\Psi_{i 0}^{+}\right\rangle \cdot{ }^{21,22}$

The rate of emission of a molecule is determined by the Einstein coefficient of spontaneous emission ${ }^{27}$

$$
A_{\mathrm{fi}}=\frac{8 \pi^{3} \nu_{\mathrm{fi}}^{3}}{3 \varepsilon_{0} c^{3} \hbar^{2}}\left|\mu_{\mathrm{fi}}\right|^{2}
$$

where $\nu_{\mathrm{fi}}$ and $\mu_{\mathrm{fi}}$ are the frequency and dipole moment of the transition respectively. The $D_{1}$ state of an alternant radical hydrocarbon will have a vanishingly small dipole moment, and hence a very slow rate of spontaneous emission. Spontaneous emission is therefore likely to be outcompeted by undesirable internal conversion to the ground state $D_{0}$. This leads to a principal design rule: ${ }^{2}$ for a radical to be emissive, it should not be an alternant hydrocarbon. ${ }^{\ddagger}$

However, by breaking alternacy symmetry through inclusion of heteroatoms, odd-membered rings, or both, it is possible for the $D_{1}$ state to become bright, and in practice the most successful optoelectronic molecules to date are usually an alternant radical 'donor' (often based on the triphenylmethyl radical) bonded to a nonalternant 'acceptor'. ${ }^{11}$ In these systems the lowest-energy excitation is usually from the HOMO of the acceptor to the SOMO of the donor, ${ }^{2}$ which we denote $\left|\Psi_{h}^{0}\right\rangle$. This would generally be expected to be dark since relevant orbitals are spatially disjoint, ${ }^{16}$ but with relevant orbital interaction it is possible for this excitation to 'borrow intensity ${ }^{16,30}$ from the intense $\left|\Psi_{10}^{+}\right\rangle$transition of the radical, ${ }^{2}$ such that the $\left|\Psi_{h}^{0}\right\rangle$ state has a dipole moment at first order

$$
\left\langle\Psi_{0}|\hat{\mu}| \Psi_{h}^{0,(1)}\right\rangle \simeq\left\langle\Psi_{0}|\hat{\mu}| \Psi_{10}^{+}\right\rangle \frac{\left\langle\Psi_{10}^{+}|\hat{H}| \Psi_{h}^{0,(0)}\right\rangle}{E\left(\Psi_{h}^{0,(0)}\right)-E\left(\Psi_{10}^{+}\right)}
$$

where $\left|\Psi_{h}^{0,(0)}\right\rangle$ and $\left|\Psi_{h}^{0,(1)}\right\rangle$ are the acceptor-HOMO to donor-SOMO excitation states perturbed to zero and first order respectively. Analysis of this expression has led to design rules based on orbital interaction. ${ }^{2,11}$

\subsection{Solvent dependence}

The absence of any dipole moment (or higher multipole) in an alternant hydrocarbon radical from Eq. (63) means that such molecules will show minimal solvatochromic shift with absorption. This would reasonably expected to hold in an amorphous film (with the radical embedded in a host material, as is commonly seen in OLEDs ${ }^{1,2}$ ), such that the film absorption is relatively insensitive to the polarizability of the host material.

This result is also advantageous from a computational perspective since, as the solvent is unlikely to substantially influence absorption spectra, the excitation energy values calculated by a conventional, gas-phase, static calculations (e.g. in Refs 1,2,16) may not need significant adjustment for solvation effects.

Whether or not a solvatochromic shift is seen in emission depends on the dipole moment of the excited state, which in turn depends on the orbital parentage of that state. A detailed analysis of this is outside the scope of the paper and left for further research.

\footnotetext{
${ }^{\ddagger}$ It may be possible to construct extremely rigid alternant radicals in which internal conversion is hindered; see Ref 11 for more details.
} 


\subsection{Non-aufbau occupancy}

Recent articles ${ }^{5,10}$ reported that certain (non-alternant) organic radicals had a non-aufbau orbital occupancy, such that the energy of the SOMO was lower than the energy of the HOMO (and in some cases, lower than the energy of other doubly-occupied orbitals), ${ }^{5}$ illustrated in Fig. 3. These articles used DFT and high-level multiconfigurational calculations to confirm their results. We can, however, provide a theoretical explanation based on the results in this paper in section 4, and which do not rely on any assumption of alternancy or PPP approximations.

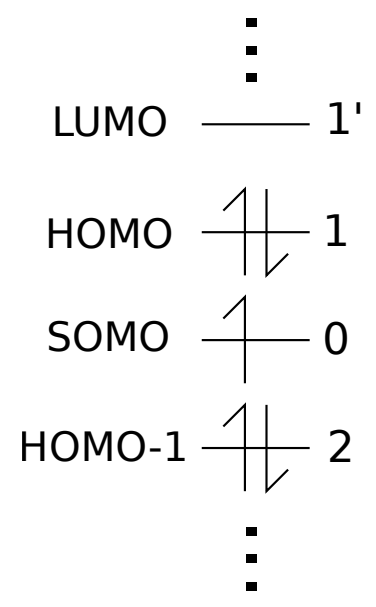

Figure 3. Schematic molecular orbital diagram of a radical molecule with a non-aufbau orbital occupancy, showing the SOMO beneath the HOMO in energy.

For a molecule to have non-aufbau occupancy, the energy of the configuration with the SOMO (orbital 0 in our notation) beneath the energy of the HOMO (orbital 1) in our notation must be of lower energy than the energy of moving an electron from the (higher-lying) HOMO to the (lower-lying) SOMO. We therefore consider a hypothetical molecule with non-aufbau occupancy for which the self-consistent field equations have been solved with electron occupancy of that in Fig. 3. We already know from Eq. (15b) that

$$
E\left(\Psi_{\overline{1}}^{\overline{0}}\right)-E_{0}=F_{00}-F_{11}-J_{10}+\frac{1}{2} K_{10}+\frac{1}{2} J_{00}
$$

and by construction we are considering a system where $F_{00}<F_{11}$. Consequently, in order for $E\left(\Psi_{\overline{1}}^{\overline{0}}\right)>E_{0}$,

$$
F_{11}-F_{00}<\frac{1}{2} J_{00}-J_{10}+\frac{1}{2} K_{10}
$$

By construction the LHS of Eq. (67) is positive, and from standard electronic structure theory ${ }^{32} J_{10}>K_{10}>0$. It does not follow that, in general, the RHS of Eq. (67) will be positive or greater than the LHS. This is the case for radicals who have an aufbau occupancy. However, if we consider a situation where the SOMO and HOMO are spatially disjoint and far separated then we can approximate $J_{00} \gg J_{10}>K_{10} \simeq 0$, then it may be possible for the RHS of Eq. (67) to be positive. This suggests that molecules where the HOMO and SOMO are spatially disjoint (such that an excitation between them would be of charge-transfer character) then it is more likely for non-aufbau character to be observed, and which is observed computationally. ${ }^{5,10}$

\section{CONCLUSIONS}

In this article we have rederived the original application of electronic structure theory to conjugated alternant organic radicals. ${ }^{21,22}$ As well as presenting previous results in modern notation we have derived further results, such as the excitation energy from orbital HOMO $-n$ to SOMO being identical to that from the SOMO to the $\mathrm{LUMO}+n$, even including two-electron (Coulomb and Exchange) effects. We have also proven that a ground-state alternant hydrocarbon radical will have no dipole or higher multipole.

These results, of course, rely on a number of approximations, such as the neglect of $(p 0 \mid 00)$ terms in Eq. (9), the use of a minimal basis set, and the neglect of differential overlap. Although arguably crude, the predicts 
of the theory (such as a very weak $D_{1}$ absorption and an intense $D_{2}$ absorption) are generally borne out by experimental results. ${ }^{2,11}$

The use of organic radicals in optoelectronics is now a rapidly increasing, but comparatively unexplored, field compared to closed-shell systems and there are therefore many avenues of future research.

From a theoretical perspective, it is probably possible to derive further results for alternant organic radicals, such as the static dipole moment of excited states, and the mixing of excitations of the form $\left|\Psi_{i}^{j^{\prime},{ }^{+}}\right\rangle$. Further results may arise from combining the results here with intensity borrowing perturbation theory, ${ }^{30}$ extending the results already obtained in Refs. 2,11. It may be possible to combine these results with pre-existing theoretical considerations such as group theory and spin selection rules to formulate further design principles.

From a computational perspective, it will be instructive to simulate radicals using the approximations given here and compare this to high-level calculations such as complete active space self-consistent field (CASSCF) calculations which have been previously used to benchmark PPP theory. ${ }^{16}$

Overall we believe that there is huge scope for theoretical principles to guide, explain and predict highly efficient optoelectronic devices.

\section{Acknowledgements}

TJHH acknowledges a Royal Society University Research Fellowship URF $\backslash$ R1 $\backslash 201502$, and thanks Emrys W. Evans for useful discussions and James D. Green for comments on the manuscript and observing the similarity between the $\left|\Psi_{i}^{j^{\prime},+}\right\rangle$ energy and the standard expression for the singlet state energy.

\section{REFERENCES}

[1] Ai, X., Evans, E. W., Dong, S., Gillett, A. J., Guo, H., Chen, Y., Hele, T. J. H., Friend, R. H., and Li, F., "Efficient radical-based light-emitting diodes with doublet emission," Nature 563, 536-540 (Nov. 2018).

[2] Abdurahman, A., Hele, T. J. H., Gu, Q., Zhang, J., Peng, Q., Zhang, M., Friend, R. H., Li, F., and Evans, E. W., "Understanding the luminescent nature of organic radicals for efficient doublet emitters and pure-red light-emitting diodes," Nature Materials 19, 1224-1229 (Nov. 2020).

[3] Cui Zhiyuan, Abdurahman Alim, Ai Xin, and Li Feng, "Stable Luminescent Radicals and Radical-Based LEDs with Doublet Emission," CCS Chemistry 2(4), 1129-1145 (2020).

[4] Hiratsuka, H., Rajadurai, S., Das, P. K., Hug, G. L., and Fessenden, R. W., "Evidence for non-radiative triplet-doublet energy transfer in the naphthalene-benzophenone system in tetrahydrofuran," Chemical Physics Letters 137, 255-260 (June 1987).

[5] Guo, H., Peng, Q., Chen, X.-K., Gu, Q., Dong, S., Evans, E. W., Gillett, A. J., Ai, X., Zhang, M., Credgington, D., Coropceanu, V., Friend, R. H., Brédas, J.-L., and Li, F., "High stability and luminescence efficiency in donor-acceptor neutral radicals not following the Aufbau principle," Nature Materials 18, 977-984 (Sept. 2019).

[6] Peng, Q., Obolda, A., Zhang, M., and Li, F., "Organic Light-Emitting Diodes Using a Neutral pi Radical as Emitter: The Emission from a Doublet," Angewandte Chemie International Edition 54(24), 7091-7095 (2015).

[7] He, C., Li, Z., Lei, Y., Zou, W., and Suo, B., "Unraveling the Emission Mechanism of Radical-Based Organic Light-Emitting Diodes," The Journal of Physical Chemistry Letters 10, 574-580 (Feb. 2019).

[8] Cho, E., Coropceanu, V., and Brédas, J.-L., "Organic Neutral Radical Emitters: Impact of Chemical Substitution and Electronic-State Hybridization on the Luminescence Properties," Journal of the American Chemical Society 142, 17782-17786 (Oct. 2020).

[9] Cho, E., Coropceanu, V., and Brédas, J.-L., "Impact of chemical modifications on the luminescence properties of organic neutral radical emitters," Journal of Materials Chemistry C (May 2021).

[10] Abella, L., Crassous, J., Favereau, L., and Autschbach, J., "Why is the Energy of the Singly Occupied Orbital in Some Radicals below the Highest Occupied Orbital Energy?," Chemistry of Materials 33, 36783691 (May 2021). 
[11] Hudson, J. M., Hele, T. J. H., and Evans, E. W., "Efficient light-emitting diodes from organic radicals with doublet emission," Journal of Applied Physics 129, 180901 (May 2021).

[12] Pariser, R. and Parr, R. G., "A semi-empirical theory of the electronic spectra and electronic structure of complex unsaturated molecules. i.," J. Chem. Phys. 21(3), 466-471 (1953).

[13] Pariser, R., "Theory of the electronic spectra and structure of the polyacenes and of alternant hydrocarbons," J. Chem. Phys. 24(2), 250-268 (1956).

[14] Pople, J. A., "Electron interaction in unsaturated hydrocarbons," Trans. Faraday Soc. 49, 1375-1385 (1953).

[15] Pople, J. A., "The electronic spectra of aromatic molecules ii: A theoretical treatment of excited states of alternant hydrocarbon molecules based on self-consistent molecular orbitals," P. Phys. Soc. Lond.. Section A 68(2), 81 (1955).

[16] Hele, T. J. H., Fuemmeler, E. G., Sanders, S. N., Kumarasamy, E., Sfeir, M. Y., Campos, L. M., and Ananth, N., "Anticipating Acene-Based Chromophore Spectra with Molecular Orbital Arguments," The Journal of Physical Chemistry A 123, 2527-2536 (Apr. 2019).

[17] Aryanpour, K., Shukla, A., and Mazumdar, S., "Theory of singlet fission in polyenes, acene crystals, and covalently linked acene dimers," J. Phys. Chem. C 119(13), 6966-6979 (2015).

[18] Jailaubekov, A. E., Willard, A. P., Tritsch, J. R., Chan, W.-L., Sai, N., Gearba, R., Kaake, L. G., Williams, K. J., Leung, K., Rossky, P. J., and Zhu, X.-Y., "Hot charge-transfer excitons set the time limit for charge separation at donor/acceptor interfaces in organic photovoltaics," Nat Mater 12, 66-73 (Jan 2013).

[19] Alvertis, A. M., Lukman, S., Hele, T. J. H., Fuemmeler, E. G., Feng, J., Wu, J., Greenham, N. C., Chin, A. W., and Musser, A. J., "Switching between Coherent and Incoherent Singlet Fission via Solvent-Induced Symmetry Breaking," Journal of the American Chemical Society 141, 17558-17570 (Nov. 2019).

[20] Longuet-Higgins, H. C., "Some Studies in Molecular Orbital Theory I. Resonance Structures and Molecular Orbitals in Unsaturated Hydrocarbons," The Journal of Chemical Physics 18, 265-274 (Mar. 1950).

[21] Dewar, M. J. S. and Longuet-Higgins, H. C., "The electronic spectra of aromatic molecules i: Benzenoid hydrocarbons," P. Phys. Soc. Lond.. Section A 67(9), 795 (1954).

[22] Longuet-Higgins, H. C. and Pople, J. A., "The Electronic Spectra of Aromatic Molecules IV: Excited States of Odd Alternant Hydrocarbon Radicals and Ions," Proceedings of the Physical Society. Section A 68, 591-600 (July 1955).

[23] Murrell, J. N. and Longuet-Higgins, H. C., "The Electronic Spectra of Aromatic Molecules III: The Effect of Inductive Substituents," Proceedings of the Physical Society. Section A 68, 329-339 (Apr. 1955).

[24] Longuet-Higgins, H. C. and Murrell, J. N., "The electronic spectra of aromatic molecules v: The interaction of two conjugated systems," P. Phys. Soc. Lond.. Section A 68(7), 601 (1955).

[25] Coulson, C. A. and Rushbrooke, G. S., "Note on the method of molecular orbitals," Math. Proc. Cambridge 36, 193-200 (4 1940).

[26] Szabo, A. and Ostlund, N., [Modern Quantum Chemistry: Introduction to Advanced Electronic Structure Theory], Dover Books on Chemistry, Dover Publications (1989).

[27] Atkins, P. W. and Friedman, R. S., [Molecular quantum mechanics], Oxford university press (2011).

[28] Evans, E. W., Olivier, Y., Puttisong, Y., Myers, W. K., Hele, T. J. H., Menke, S. M., Thomas, T. H., Credgington, D., Beljonne, D., Friend, R. H., and Greenham, N. C., "Vibrationally Assisted Intersystem Crossing in Benchmark Thermally Activated Delayed Fluorescence Molecules," The Journal of Physical Chemistry Letters 9, 4053-4058 (July 2018).

[29] Shaikh, J., Congrave, D. G., Forster, A., Minotto, A., Cacialli, F., Hele, T. J. H., Penfold, T. J., Bronstein, H., and Clarke, T. M., "Intrinsic photogeneration of long-lived charges in a donor-orthogonal acceptor conjugated polymer," Chemical Science 12, 8165-8177 (June 2021).

[30] Robinson, G. W., "Intensity enhancement of forbidden electronic transitions by weak intermolecular interactions," J. Chem. Phys. 46(2), 572 (1967).

[31] Mataga, N. and Nishimoto, K., "Electronic structure and spectra of nitrogen heterocycles," Z. Phys. Chem. 13(3), 140-157 (1957).

[32] Roothaan, C. C. J., "New Developments in Molecular Orbital Theory," Reviews of Modern Physics 23, 69-89 (Apr. 1951). 